\title{
Fos Imaging Reveals Differential Patterns of Hippocampal and Parahippocampal Subfield Activation in Rats in Response to Different Spatial Memory Tests
}

\author{
Seralynne D. Vann, ${ }^{1}$ Malcolm W. Brown, ${ }^{2}$ Jonathan T. Erichsen, ${ }^{3}$ and John P. Aggleton ${ }^{1}$ \\ ${ }^{1}$ School of Psychology, Cardiff University, Cardiff, CF10 3YG, United Kingdom, ${ }^{2}$ Department of Anatomy, University of \\ Bristol, Medical School, Bristol, BS8 1TD, United Kingdom, and ${ }^{3}$ Department of Optometry and Vision Sciences, Cardiff \\ University, Cardiff CF10 3YJ, United Kingdom
}

We compared neuronal activation, as measured by Fos staining, during different spatial tasks in two experiments. The counts of Fos-stained neurons in the hippocampus increased as the spatial demands of the tasks increased, the tasks having been carefully matched for other factors. In Experiment 1, matched groups of rats either ran a standard eight-arm radial maze task or were trained to run up and down just one arm of the maze; the number of runs and rewards was identical in both conditions. In Experiment 2, rats were trained on the eight-arm maze but in different rooms. On the critical test day, both groups were run in the same room so that one group now performed with novel landmarks. All hippocampal subfields (dentate gyrus, CA3, CA1, dorsal, ventral, and caudal subiculum) showed a relative increases in c-fos activation in the eight-arm (Experiment 1) and novel room (Experiment 2) conditions, the sole exception being the ventral subiculum in Ex- periment 2. Although increased c-fos activation was found in both dorsal and ventral hippocampus, in Experiment 2 the relative increase was significantly greater in the dorsal hippocampus. Parahippocampal cortices responded heterogeneously: the perirhinal cortex failed to show increased activation in both experiments, in contrast to the entorhinal and postrhinal cortices. Subsequent comparisons confirmed that the perirhinal and postrhinal cortices responded in qualitatively different ways, the perirhinal cortex differing from the rest of the hippocampal formation. These experiments, which provide the first analysis of hippocampal Fos production during tests of allocentric spatial working memory, reveal that all components of the hippocampus are activated, but that under certain conditions the dorsal hippocampus is disproportionately involved.

Key words: Fos; spatial memory; hippocampus; entorhinal cortex; dorsal hippocampus; parahippocampal cortex
The importance of the rodent hippocampus for spatial memory has been clearly demonstrated by lesion and by single-cell recording studies (O'Keefe and Nadel, 1978; Morris et al., 1982). Although these approaches have revealed much about spatial processing in the hippocampus, they have inherent limitations. These include the difficulty of adequately sampling neuronal populations in multiple, defined brain regions [but see Jung et al. (1994)] and, in the case of lesion studies, the fact that the analyses always involve the functioning of an abnormal brain. Expression of the Fos gene is an indirect correlate of increased neuronal activity (Sagar et al., 1988; Dragunow and Faull, 1989; Herrera and Robertson, 1996) and has repeatedly been shown to be induced under conditions of learning (Herdegen and Leah, 1998; Tischmeyer and Grimm, 1999). It can therefore be used to detect differential activation in specific brain sites in the intact brain. Potential limitations of this method include the fact that c-fos is not expressed in every brain region (Chaudhuri, 1997), and so we focused on relative changes in sites that do express this gene. To explore spatial memory we measured the differential activation of c-fos in specific hippocampal subfields and related cortical regions

\footnotetext{
Received Sept. 23, 1999; revised Jan. 13, 2000; accepted Jan. 14, 2000.

This research was supported by a programme grant from the Medical Research Council (35 42994). We thank Alison Baird, Jo Oswald, and Clea Warburton for their assistance.

Correspondence should be addressed to Prof. J. P. Aggleton, School of Psychology, Cardiff University, Tower Building, Park Place, Cardiff, CF10 3YG, UK. E-mail: Aggleton@cardiff.ac.uk.

Copyright (C) 2000 Society for Neuroscience $\quad 0270-6474 / 00 / 202711-08 \$ 15.00 / 0$
}

during tests of spatial memory (radial arm maze) that are sensitive to hippocampal damage (Olton et al., 1979).

The present study had several related goals. The first was to compare hippocampal activity during tasks that differed systematically in their demands on spatial memory but were matched for visual, motor, and somatosensory demands. Although a previous study reported hippocampal c-fos activation during a spatial memory task (T-maze alternation), the comparison condition was remaining in the home cage (Nagahara and Handa, 1995) and hence was not an adequate control. Thus, in Experiment 1, the comparison was between matched pairs of animals performing either a standard radial arm maze task or the same number of runs in just one of the arms of the same maze, for the same number of food rewards. In Experiment 2, matched pairs of animals were trained on the standard radial arm maze task but consistently in one or the other of two different rooms. On the critical test day the maze was used by all of the rats in only one of these rooms, so that half the rats had to learn a new array of spatial landmarks while performing the task. It was predicted that this new room condition would produce greater hippocampal activation because the rats had to learn new landmarks and remember their spatial choices. In contrast, the one-arm condition in Experiment 1 would produce the least activation because there was no explicit spatial memory component, although all other demands were similar.

The second goal was to compare dorsal/ventral activation within the hippocampus during all of these spatial tasks. From an analysis of selective lesions, single-unit recordings, and a consid- 
eration of the anatomy, it has been proposed that the dorsal hippocampus of the rat is the more critical for spatial learning (Moser et al., 1993, 1995; Jung et al., 1994; Hock and Bunsey, 1998; Moser and Moser, 1998a,b). A direct prediction is that dorsal hippocampus will show greater activation during spatial tasks. The third goal was to compare Fos production in the parahippocampal region (the entorhinal, perirhinal, and postrhinal cortices). This region is the principal source of cortical information reaching the hippocampus, and its functions are regarded as being tightly linked to those of the hippocampus (Witter et al., 1989; Eichenbaum et al., 1994; Burwell et al., 1995), yet there is conflicting evidence about the importance of its subfields for spatial processing (Kolb et al., 1994; Wiig and Bilkey, 1994; Otto et al., 1997; Bussey et al., 1999). Thus, the goal of this study was to examine the involvement of different components of the hippocampal formation in spatial memory using a noninvasive technique with a high degree of spatial resolution. To minimize the impact of nonspatial processes, the study used matched pairs of behavioral tasks that differed in their spatial demands.

\section{MATERIALS AND METHODS}

\section{Subjects}

Subjects were 24 male pigmented rats (DA strain; Harlan) weighing from 175 to $220 \mathrm{gm}$. They were food-deprived to $85 \%$ of their free-feeding body weight and maintained at this level throughout the experiment. Water was available ad libitum. Animals were caged in pairs, and these became the matched pairs with one from each pair being placed in each treatment group. Before the study the animals were thoroughly habituated to handling.

\section{Apparatus}

Testing was performed in an eight-arm radial maze. The maze consisted of an octagonal central platform (34 $\mathrm{cm}$ diameter) and eight equally spaced radial arms ( $87 \mathrm{~cm}$ long, $10 \mathrm{~cm}$ wide). The base of the central platform and the arms were made of wood, and clear Perspex $(24 \mathrm{~cm}$ high) formed the walls of the arms. At the end of each arm was a food well $2 \mathrm{~cm}$ in diameter and $0.5 \mathrm{~cm}$ deep. At the start of each arm was a clear Perspex guillotine door $12 \mathrm{~cm}$ high that controlled access in and out of the central platform. Each door was attached to a pulley system that enabled the experimenter to control access to the arms.

All animals in Experiment 1 were tested in the same rectangular room $(295 \times 295 \times 260 \mathrm{~cm})$, which contained salient visual cues such as geometric shapes and high-contrast stimuli. Half of the animals in Experiment 2 were trained in a second room, which differed in its overall shape, size $(255 \times 330 \times 260 \mathrm{~cm})$, lighting, position of the experimenter, and visual cues placed on the walls.

\section{Behavioral training}

Experiment 1. One of the animals in each of six matched pairs (Group 8 arm-1) was trained to run in the maze using a standard working memory procedure (Olton et al., 1978). Thus at the start of a trial all eight arms were baited with a single food pellet $(45 \mathrm{mg}$; Noyes Purified Rodent Diet). When the rat returned to the central platform, all doors were closed for $\sim 5 \mathrm{sec}$ before they were again opened, permitting the animal to make a choice. This continued until all eight arms had been visited. Retrieving all eight pellets constituted a single trial, composed of eight or more arm runs. Training continued until the animals could reliably retrieve all eight pellets without making an error (i.e., not visit an arm that had already been entered on that trial); this typically required between seven and nine sessions. The only noteworthy aspect of the training was that each session consisted of multiple trials in the radial arm maze, one after the other, so that each session lasted for $30 \mathrm{~min}$ to prolong exposure to task demands. The delay between each trial ( $2 \mathrm{~min})$ was the time it took to rebait all of the arms, and during this period the animals were placed in a traveling box that had an aluminum top, base, and sides $(10 \times 10 \times 26 \mathrm{~cm})$.

For the other six animals (Group 1arm), all arms of the radial arm maze except one were blocked off. The animal was then trained to run up and down the open arm to retrieve single pellets. The central door was opened and closed as for the other group. The number of rewards and the number of arm runs were carefully balanced across matched pairs of animals. Thus if an animal made an error in the working memory version, its partner would receive no reward on the corresponding run down the single arm. This animal was also placed in the aluminum traveling box after performing the same number of arm runs as its partner for each trial, and again it was left there for 2 min before being returned to the central platform for the next trial.

Final session. The final session was the same as those in training, i.e., 30 min of radial arm maze testing (approximately seven radial arm maze trials) or a matched number of runs down a single arm. After completion of testing, each animal was placed in a soundproof box in a dark, quiet room for $90 \mathrm{~min}$. The animals had been habituated to this post-training procedure after all preceding sessions.

Experiment 2. Animals were trained to run the standard radial arm maze task in one of two rooms, but the same apparatus was used throughout the study so that only extra-maze cues distinguished the rooms. The six animals in Group 8arm-2 were trained and tested in exactly the same way as those in Experiment 1 (8arm-1), using the same room and maze. The six matched animals in Group 8arm-novel also received the same protocol, but during training the maze was placed in a room with very different spatial landmarks.

Final session. The final session for Group 8arm-2 was identical to that used for the comparable group in Experiment 1 (Group 8arm-1). For Group 8arm-novel the animals were tested, for the first time, in the same room as that used by Group 8arm-2. Both groups performed the radial arm maze task between seven and nine times. As in Experiment 1, the animals were placed in a soundproof box in a dark, quiet room for $90 \mathrm{~min}$ after testing.

\section{Immunocytochemistry}

Ninety minutes after running the radial arm maze, the animals were deeply anesthetized with sodium pentobarbital $(1 \mathrm{mg} / \mathrm{kg})$ and perfused transcardially with $0.1 \mathrm{M}$ PBS followed by $4 \%$ paraformaldehyde in $0.1 \mathrm{M}$ PBS. The brains were removed and post-fixed in the $4 \%$ paraformaldehyde for $4 \mathrm{hr}$ and then transferred to $30 \%$ sucrose overnight at $4^{\circ} \mathrm{C}$. Coronal sections were cut at $30 \mu \mathrm{m}$ on a freezing microtome, and a one in two series was collected in $0.1 \mathrm{M}$ PBS containing $0.2 \%$ Triton X-100 (PBST). A peroxidase block was then performed in which the sections were transferred to $0.3 \%$ hydrogen peroxide in PBST for $10 \mathrm{~min}$ to inhibit endogenous peroxidase and then washed several times with PBST. Sections were incubated in PBST containing Fos rabbit polyclonal antibody (1:5000; Ab-5, Oncogene Science) for $48 \mathrm{hr}$ at $4^{\circ} \mathrm{C}$ with periodic rotation. Sections were then washed with PBST and incubated in biotinylated goat anti-rabbit secondary antibody (diluted 1:200 in PBST; Vectastain, Vector Laboratories, Burlingame, CA) and 1.5\% normal goat serum for $2 \mathrm{hr}$ at room temperature on a rotator. Sections were then washed and processed with avidin-biotinylated horseradish peroxidase complex in PBST (Elite Kit, Vector Laboratories) for $1 \mathrm{hr}$ at room temperature, again with constant rotation. Sections were washed again in PBST and then in $0.05 \mathrm{M}$ Tris buffer. The reaction was then visualized using diaminobenzidine (DAB Substrate Kit, Vector Laboratories). The reaction was stopped by washing in cold PBS, and then sections were mounted on gelatin-coated slides, dehydrated through a graded series of alcohols, and coverslipped. One in four sections was mounted directly onto slides and stained using cresyl violet, a Nissl stain, for histological identification of specific brain regions.

\section{Regions of interest}

Cytoarchitectonic subfields within the hippocampal formation were identified from coronal sections, using the nomenclature of Swanson (1992). These consisted of the dentate gyrus (DG), CA3, and CA1, and the dorsal, ventral, and caudal subiculum (see Fig. 1). The "dorsal" and "ventral" hippocampal counts were taken from the same coronal slices and corresponded to anteroposterior (AP) level $-5.0 \mathrm{~mm}$ relative to bregma in Swanson (1992). The border between these two regions (see Fig. 1) corresponded to dorsoventral level $-5.0 \mathrm{~mm}$ from bregma (Moser et al., 1995). The dorsal and ventral hippocampal counts involved just the DG and fields CA1 and CA3, i.e., not the subiculum complex. At this level the dentate gyrus is present in both the dorsal and ventral hippocampus.

We also counted Fos-reactive cells in the parahippocampal region (Witter et al., 1989; Burwell et al., 1995). The perirhinal counts involved both areas 35 and 36 (Burwell et al., 1995), whereas the postrhinal cortex only involved cortex posterior to the perirhinal cortex and dorsal to the rhinal sulcus [corresponding to the ectorhinal area in Swanson (1992) 
and Burwell and Amaral (1998)]. The lateral and medial entorhinal cortices were considered separately in light of their different connection patterns (Witter et al., 1989; Naber et al., 1997).

In addition, we examined activation in three cortical control sites. These sites, the visual cortex (primary visual area; VISp), the somatosensory cortex (primary somatosensory area; SSp), and the motor cortex (primary motor area; MOp) were selected because if the behavioral tasks have been appropriately matched for nonspatial demands they would be expected to show no differences. The counts for these regions were taken across all cortical layers.

\section{Image analysis}

Sections were scanned using a Leitz Diaplan microscope equipped with a Dage MTI CCD72S camera interfaced to a Power Macintosh computer $(8500 / 150)$ by a Scion LG-3 frame-grabber board. After image processing, counts of the stained nuclei were performed using the public domain $\mathrm{NIH}$ Image program. Cortical areas were assessed using counts above threshold in a standard frame sample area $(0.78 \times 0.55 \mathrm{~mm})$ using a $10 \times$ objective. For dorsal and ventral hippocampal counts and for hippocampal subfield (dentate gyrus, CA3, and CA1) counts, the entire extent of the target region within the selected coronal sections was assessed (see Fig. 1). For all brain areas analyzed, counts were taken from at least four consecutive sections across both hemispheres, and these counts were averaged to produce a mean.

Counts were normalized to reduce variability across matched pairs of animals. This was done by dividing the mean number of activated neurons in a given animal for a given site by the combined mean of the two animals in each matched pair and expressing this result as a percentage. Thus all normalized scores across pairs sum to 100. These normalized data were then used for the statistical analyses. Matched $t$ tests were used to compare the cortical control sites (to minimize type II errors), whereas hippocampal regions were analyzed in an overall analysis of variance with two factors: spatial condition and brain region. When appropriate, the simple effects for each brain region were analyzed as recommended by Winer (1971). The data from the hippocampal subfields and parahippocampal regions were grouped separately before being analyzed in separate ANOVAs.

\section{RESULTS}

\section{Behavioral results}

On the final test day of Experiment 1, half the animals performed a standard version of the eight-arm radial maze. Testing took place over a 30 min session, and animals typically performed a total of seven trials (retrieval of all eight pellets) in this session. The mean number of errors per trial across all trials within this session ( \pm SEM in parentheses) was $1.1(0.2)$, and the mean number of correct responses in the first eight choices was 7.2 (0.1). The control animals (Group 1arm) received exactly the same number of arm runs, rewards, and errors.

On the final test day of Experiment 2, all animals performed the radial arm maze task in the same room, but for one set of animals the room was novel. Although the 8 arm-novel rats were slower to complete the first trial $\left(t_{(10)}=2.42, p<0.05\right)$, they quickly speeded up so that by the end of the session the two groups were indistinguishable (for last trial, $t<1$ ). Most importantly, there were no differences in the accuracy levels of the two groups of animals from the very first trial as measured by total errors $(t<1)$ or total correct in first eight choices $\left(t_{(10)}=1.1, p>\right.$ 0.2 ). Similarly there was no overall group difference when all trials within the final session were summed (mean errors per trial, $t_{(10)}=1.50, p>0.1$; mean correct in first eight choices, $t<1$ ). An analysis of the sequence of arms selected by the rats (Ennaceur and Aggleton, 1997) in both experiments provided no evidence that the rats were using a simple egocentric strategy (e.g., always turn to the right) to solve the radial arm maze task.
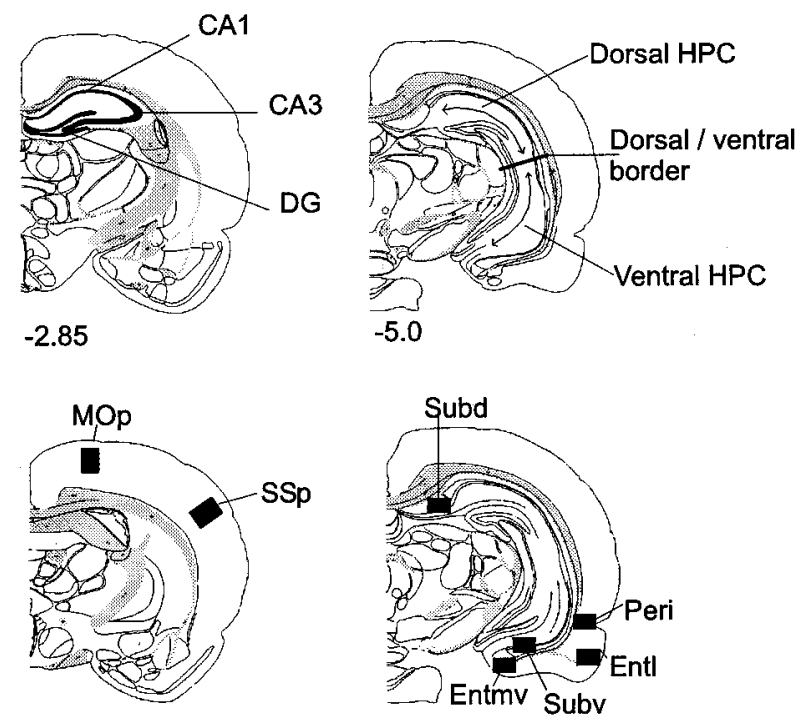

$-1.53$
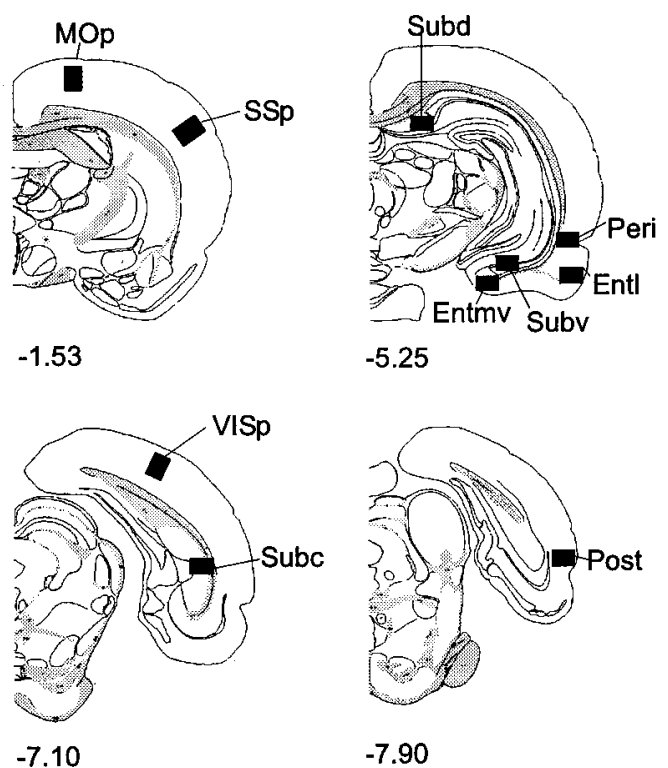

$-7.10$

$-7.90$

Figure 1. Diagrams of coronal sections indicating areas sampled. The numbers indicate the distance (in millimeters) of the sections from bregma (Swanson, 1992). DG, Dentate gyrus; Dorsal HPC, dorsal hippocampus; Entl, lateral entorhinal cortex; Entmv, medial entorhinal cortex; $M O p$, primary motor cortex; Peri, perirhinal cortex; Post, postrhinal cortex; $S u b c$, caudal subiculum; $S u b d$, dorsal subiculum; $S u b v$, ventral subiculum; $S S p$, primary somatosensory cortex; VISp, primary visual cortex; Ventral $H P C$, ventral hippocampus.

\section{Fos counts}

\section{Control regions}

The control areas that were examined were the SSp, MOp, and VISp (Fig. 1). There was no evidence of a difference in c-fos activation between the paired groups of animals in either Experiment 1 or 2 for any of the three control regions (all comparisons $t<1$ ) (Fig. 2).

\section{Dorsal versus ventral hippocampus}

The dorsal and ventral counts were taken from the same coronal level and involved the corresponding portions of the dentate gyrus, CA1, and CA3. Before directly comparing dorsal with ventral hippocampus, we tested for differences across conditions within the dorsal and ventral hippocampus. Counts of nuclei stained for Fos were significantly higher in both the dorsal and ventral hippocampus in the more spatially demanding conditions in both experiments. Thus in Experiment 1 there were highly significant differences for 8 arm-1 versus 1-arm (dorsal hippocampus $F_{(1,17)}=174.1, p<0.001$; ventral hippocampus $F_{(1,17)}=$ 136.0, $p<0.001)$. A similar effect was observed in Experiment 2 with the 8arm-novel condition resulting in the greatest c-fos activation (dorsal hippocampus $F_{(1,17)}=90.1, p<0.001$; ventral hippocampus $\left.F_{(1,17)}=11.0, p<0.005\right)$. 

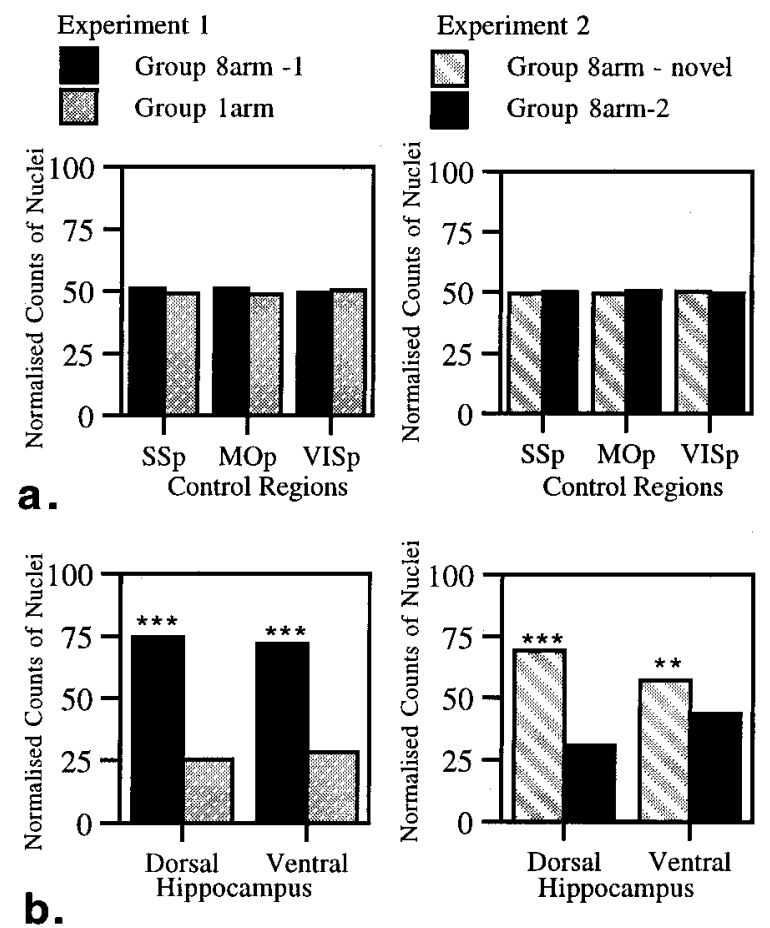

Figure 2. $a$, Normalized counts of Fos-stained nuclei for control regions in Experiments 1 and 2. $b$, Normalized counts of Fos-stained nuclei for dorsal and ventral hippocampus in Experiments 1 and 2. Data are shown as means $\pm \mathrm{SE}$; where SE is very small, they are not visible on the graphs. All normalized scores sum to 100 (see Materials and Methods). See Figure 1 for abbreviations. Significance of differences in normalized counts: ${ }^{* *} p<0.005,{ }^{* * *} p<0.001$.

The critical comparisons concerned the relative increase in activation across the dorsal and ventral hippocampus in the paired conditions. In Experiment 1 the relative increase in stained nuclei was similar for both dorsal and ventral hippocampus (Fig. 2), and as a consequence, the interaction was not significant $\left(F_{(1,10)}=0.83\right)$. Experiment 2 produced a different pattern of results because the animals tested in the novel room showed a greater enhancement of c-fos activation in the dorsal hippocampus. This was confirmed by the significant interaction between dorsal and ventral hippocampal counts $\left(F_{(1,10)}=31.4\right.$, $p<0.0005)$. The qualitative difference in the patterns of dorsal/ ventral activation across the two experiments was underlined by a significant three-way interaction from the ANOVA using data from both sets of experiments $\left(F_{(1,20)}=6.34, p<0.05\right)$.

\section{Hippocampal subfields}

The next group of structures to be compared were the hippocampal subfields: dentate gyrus, CA3, CA1, dorsal subiculum, ventral subiculum, and caudal subiculum (Fig. 1). In both experiments the more spatially demanding condition resulted in higher levels of Fos. In Experiment 1 the counts of stained nuclei were higher for all the above subregions when the animals performed the standard version of the radial arm maze compared with those animals that ran up and down only one arm of the maze (Fig. 3, Experiment 1). This was shown by a main effect of condition $\left(F_{(1,}\right.$ $10)=95.4, p<0.0001)$. Further analyses revealed that this difference was significant for every subregion that had been counted (all $p<0.001$ ).

Performing the maze task in a novel room (Experiment 2) also resulted in an increase in stained nuclei across the subregion as shown by the significant effect of condition $\left(F_{(1,10)}=36.6, p<\right.$ $0.0005)$. This difference was significant for all subregions except for the ventral subiculum $\left(\mathrm{DG} F_{(1,31)}=20.9, p<0.001\right.$; CA1 $F_{(1,31)}=62.1, p<0.001$; dorsal subiculum $F_{(1,31)}=30.4, p<$ 0.001 ; caudal subiculum $F_{(1,31)}=15.9, p<0.001$; CA3 $F_{(1,31)}=$ $7.4, p<0.05$; ventral subiculum $\left.F_{(1,31)}=3.0, p=0.09\right)$.

In view of the results for the separate subfields and the interaction between the dorsal and ventral hippocampus, we examined whether the relative increase in dorsal as compared with ventral counts in Experiment 2 was found across the three subfields (DG, CA3, and CA1) to the same extent. Using dorsal and ventral subfield counts taken from the same coronal sections, we performed a three-way ANOVA. The lack of an interaction between these factors $\left(F_{(2,40)}=1.13, p>0.1\right)$ showed that the dorsal increases in activation were similar across all subfields, i.e., all three subfields contributed to the dorsal hippocampal enhancement effect. These findings prompted a comparison between the dorsal and ventral subiculum. A significant interaction between these two subiculum regions was found in both Experiment 1 $\left(F_{(1,10)}=9.94, p<0.05\right)$ and Experiment $2\left(F_{(1,10)}=27.8, p<\right.$ $0.0005)$. There was no three-way interaction in this case, however, showing that the nature of the increase across the subiculum was similar in both experiments. Thus, unlike the other hippocampal subfields, the subiculum showed a dorsal enhancement effect in both Experiments 1 and 2.

\section{Parahippocampal cortices}

Counts were made in the medial and lateral entorhinal cortices, the perirhinal cortex, and the postrhinal cortex. In Experiment 1 there was a significant effect of condition $\left(F_{(1,10)}=60.7, p<\right.$ $0.0001)$ because higher numbers of Fos-positive cells were found in the animals performing the standard radial arm maze task. Subsequent analyses showed highly significant differences in the lateral entorhinal, the medial entorhinal, and the postrhinal cortices (lateral entorhinal $F_{(1,39)}=11.5, p<0.005$; medial entorhinal $F_{(1,39)}=21.5, p<0.0001$; postrhinal $F_{(1,39)}=25.7, p<$ $0.001)$. In striking contrast, no difference was found in the perirhinal cortex $(F<1)$.

A similar pattern was found in Experiment 2: there was a highly significant effect of condition $\left(F_{(1,10)}=101.7, p<0.0001\right)$, and the same three regions showed significantly greater activation in those animals performing in a novel room (lateral entorhinal $F_{(1,37)}=10.3, p<0.005$; medial entorhinal $F_{(1,37)}=19.7, p<$ 0.001 ; postrhinal $\left.F_{(1,37)}=28.3, p<0.001\right)$. Once again, the perirhinal counts were an exception because they failed to differ significantly $\left(F_{(1,37)}=3.08, p=0.09\right)$.

A consistent feature of both experiments was the increase in entorhinal and postrhinal activation that contrasted with the lack of a difference in the perirhinal cortex. To compare more directly the postrhinal and perirhinal results, we looked at the group by region interactions for these two sites. Both experiments showed a significant interaction between these two regions $\left(F_{(1,10)}=\right.$ 8.47, $p<0.05 ; F_{(1,10)}=14.3, p<0.005$ for Experiments 1 and 2, respectively). These interactions reflect the relatively greater increase in activation in the postrhinal cortex in the more spatially demanding conditions, as compared with the perirhinal cortex, which had similar, moderate levels of activation across both experiments. In Experiment 1 there was also a significant interaction between lateral and medial entorhinal cortex and perirhinal cortex (lateral entorhinal and perirhinal $F_{(1,10)}=16.5, p<$ 0.005 ; medial entorhinal and perirhinal $\left.F_{(1,10)}=9.3, p<0.05\right)$. 
Experiment 1

Group 8arm-1 Group larm
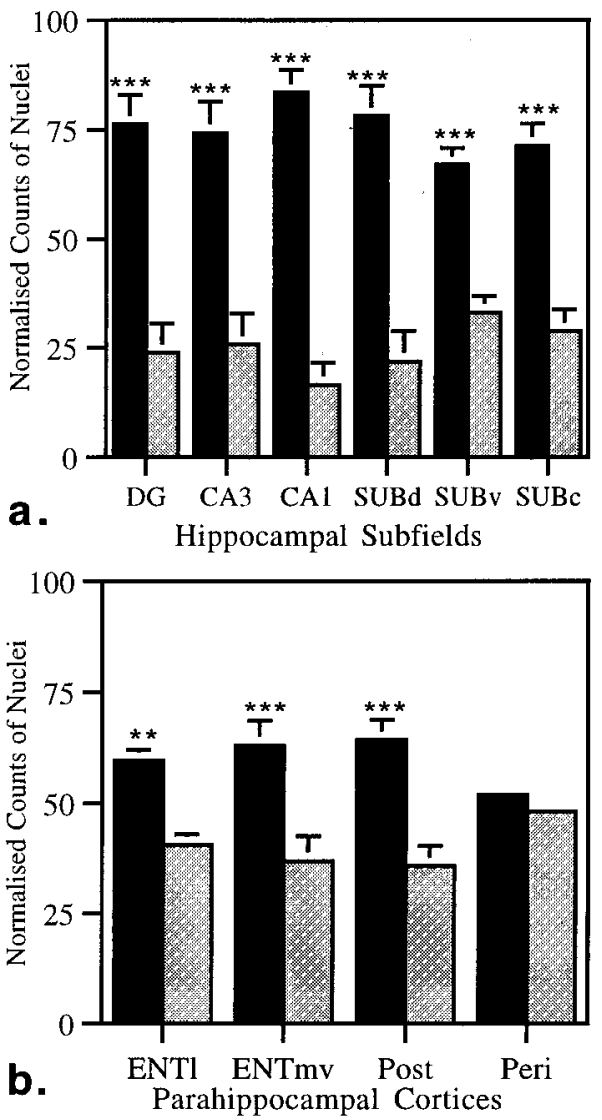

Experiment 2
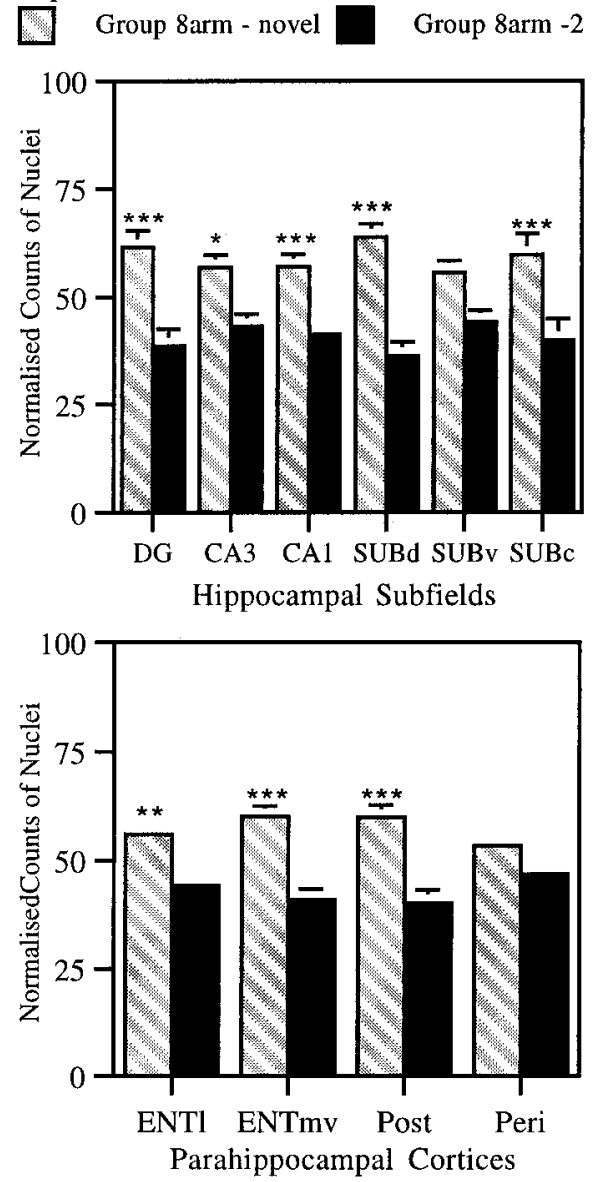

Figure 3. $a$, Normalized counts of Fosstained nuclei for hippocampal subfields in Experiments 1 and 2. $b$, Normalized counts of Fos-stained nuclei for parahippocampal cortices in Experiments 1 and 2. Data are shown as means $\pm \mathrm{SE}$; where SE is very small, they are not visible on the graphs. All normalized scores sum to 100 (see Materials and Methods). See Figure 1 for abbreviations. Significance of differences in normalized counts: ${ }^{*} p<0.05,{ }^{* *} p<0.005,{ }^{* * *} p<$ 0.001 .
These interactions were not significant in Experiment $2\left(F_{(1,10)}=\right.$ $2.77, p>0.1 ; F_{(1,10)}=2.30, p>0.1$, respectively).

To see how closely parahippocampal counts mirrored the counts for the hippocampus proper, we compared the counts for the four parahippocampal regions with a "total" hippocampus proper count (the combined dorsal and ventral hippocampus counts for DG, CA1, and CA3) as a way of looking at the relative increase. In Experiment 1, each of the four regions showed significant interactions with the total hippocampus proper (lateral entorhinal cortex $F_{(1,10)}=22.2, p<0.001$; medial entorhinal cortex $F_{(1,10)}=8.10, p<0.05$; postrhinal cortex $F_{(1,10)}=10.3$, $p<0.01$; perirhinal cortex $\left.F_{(1,10)}=47.08, p<0.0001\right)$. These interactions reflected the finding that for the parahippocampal regions there was not as great an enhancement of c-fos activation in the standard radial arm maze task as in the hippocampus proper. Experiment 2 revealed a different picture: the perirhinal cortex was now the only parahippocampal region that showed a significant interaction with total hippocampus proper counts $\left(F_{(1,10)}=9.23, p<0.05\right)$. This change in activation pattern for the entorhinal and postrhinal cortices was confirmed by a three-way interaction showing how, relative to the hippocampus proper, the extent of c-fos activation was task dependent (lateral entorhinal cortex $F_{(1,20)}=4.81, p<0.05$; medial entorhinal cortex $F_{(1,20)}=$ 4.14, $p<0.055$; postrhinal cortex $\left.F_{(1,20)}=6.91, p<0.05\right)$. Therefore the medial and lateral entorhinal cortices and postrhinal cortex behaved in a quantitatively similar manner to the hippocampus proper only in Experiment 2, i.e., in the more demanding task.

\section{Raw counts of nuclei}

To show the actual numbers of stained nuclei observed in each brain region examined, the means and standard errors for raw scores are presented in Tables 1 (Experiment 1) and 2 (Experiment 2), and examples of staining levels are shown in Figure 4. Analyses using the raw scores produced a general pattern very similar to that obtained with the normalized scores. Thus, out of a total of 36 analyses reported for Experiments 1 and 2, the results of only four differ. In all four cases, although the differences in the number of stained nuclei was still in the same direction, the result no longer reached the 0.05 level of significance (Experiment 1, CA3 and medial entorhinal cortex; Experiment 2, CA3 and caudal subiculum). This similarity between the results supports the validity of using the normalized data where there is the added benefit of reducing the variance across the matched pairs of animals.

\section{DISCUSSION}

The present study used a noninvasive technique to look at the differential involvement of specific hippocampal regions during tasks that tax allocentric spatial memory. In both experiments, highly significant increases in c-fos activation were found in all hippocampal subfields (except ventral subiculum in Experiment 2) in those conditions that were more demanding on spatial memory processing, i.e., eight-arm maze versus one-arm maze (Experiment 1) and eight-arm maze in novel room versus eightarm maze in familiar room (Experiment 2). These particular tasks are assumed to tax allocentric processing because the se- 
Table 1. Raw counts of nuclei for Experiment 1

\begin{tabular}{lcc} 
Area & Group 1arm & Group 8arm-1 \\
\hline VISp & $111.9 \pm 27.2$ & $119.4 \pm 32.0$ \\
MOp & $74.4 \pm 13.9$ & $74.9 \pm 17.3$ \\
SSp & $109.1 \pm 30.5$ & $108.6 \pm 31.6$ \\
Peri & $62.1 \pm 12.1$ & $68.4 \pm 14.6$ \\
Post & $42.6 \pm 14.3$ & $62.4 \pm 15.8$ \\
Entl & $18.4 \pm 6.0$ & $26.3 \pm 7.1$ \\
Entmv & $34.8 \pm 12.1$ & $47.9 \pm 8.9$ \\
Subd & $9.1 \pm 4.5$ & $28.6 \pm 8.3$ \\
Subv & $16.4 \pm 4.07$ & $27.9 \pm 4.2$ \\
Subc & $14.7 \pm 5.8$ & $28.4 \pm 6.5$ \\
DG & $2.6 \pm 0.8$ & $8.0 \pm 2.5$ \\
CA3 & $2.6 \pm 0.9$ & $7.0 \pm 2.8$ \\
CA1 & $1.3 \pm 0.7$ & $4.1 \pm 1.0$ \\
Dorsal HPC & $7.8 \pm 2.2$ & $21.8 \pm 4.6$ \\
Ventral HPC & $27.6 \pm 4.9$ & $66.1 \pm 3.5$
\end{tabular}

The mean counts \pm SE of the number of Fos-stained nuclei for the given brain regions in Experiment 1. For areas VISp down to Subc, these numbers are taken from the standard frame sample area, which is equivalent to $0.43 \mathrm{~mm}^{2}$ (see Materials and Methods). For the remaining areas the counts are for the entire extent of the target region within the selected coronal sections. See Figure 1 for abbreviations.

\section{Table 2. Raw counts of nuclei for Experiment 2}

\begin{tabular}{lcc} 
Area & Group $8 \mathrm{arm}-2$ & Group 8arm-novel \\
\hline VISp & $215.5 \pm 13.9$ & $220.7 \pm 18.8$ \\
MOp & $169.0 \pm 14.0$ & $165.7 \pm 17.5$ \\
SSp & $180.0 \pm 14.0$ & $178.6 \pm 13.8$ \\
Peri & $136.0 \pm 15.9$ & $155.8 \pm 16.5$ \\
Post & $117.0 \pm 13.1$ & $172.1 \pm 12.5$ \\
Entl & $59.4 \pm 6.5$ & $80.2 \pm 9.8$ \\
Entmv & $108.3 \pm 10.6$ & $160.2 \pm 19.8$ \\
Subd & $46.9 \pm 6.8$ & $87.3 \pm 18.4$ \\
Subv & $57.3 \pm 6.6$ & $71.1 \pm 10.7$ \\
Subc & $52.2 \pm 7.4$ & $78.6 \pm 9.6$ \\
DG & $10.5 \pm 4.0$ & $17.1 \pm 2.7$ \\
CA3 & $12.7 \pm 2.5$ & $17.0 \pm 3.2$ \\
CA1 & $9.6 \pm 1.6$ & $21.9 \pm 3.3$ \\
Dorsal HPC & $39.6 \pm 5.6$ & $91.3 \pm 13.2$ \\
Ventral HPC & $108.7 \pm 10.4$ & $149.6 \pm 21.4$
\end{tabular}

The mean counts \pm SE of the number of Fos-stained nuclei for the given regions in Experiment 2. For areas VISp down to Subc, these numbers are taken from the standard frame sample area, which is equivalent to $0.43 \mathrm{~mm}^{2}$ (see Materials and Methods). For the remaining areas the counts are for the entire extent of the target region within the selected coronal sections. The mean counts for Experiment 2 are approximately twice those in Experiment 1. This general increase in staining is attributable to the use of a different batch of primary antibody. See Figure 1 for abbreviations.

quence of arm choices provided no evidence that an egocentric strategy was used. Furthermore, the use of multiple trials within a session would rapidly minimize the value of any intramaze odor trail cues. Last, rats of the same strain tested in the same apparatus and the same room have been demonstrated to use allocentric cues to solve this task (Bussey et al., 1999). Importantly, the comparison conditions in both experiments were matched in terms of number of runs down the arms, rate of achieving rewards, and exposure to intramaze cues. Consistent with this, there was no evidence that any of the control sites (primary motor, somatosensory, and visual cortical cortices) differed among the groups. Although previous immediate early gene stud- ies have also reported patterns of hippocampal activation that are task dependent (Bertaina and Destrade, 1995; Hess et al., 1995a,b; Nagahara and Handa, 1995), the comparisons in those cases were made between conditions that were largely uncontrolled for sensorimotor differences.

A significant increase in c-fos activation was found in both dorsal and ventral hippocampus in the more spatially demanding tasks in both experiments. Although these increases were comparable in Experiment 1, the dorsal hippocampus showed a significantly greater enhancement in Experiment 2. Subsequent analyses showed that this dorsal enhancement was found in DG, CA3, CA1, and dorsal subiculum, and thus was not confined to a particular subfield. These results reveal that there is quite a different pattern of activation when animals have to learn new landmarks with which to perform spatial working memory tasks. An intrinsic part of this novel room condition is likely to be an increase in arousal and attention as rats are confronted with unfamiliar stimuli, and this may well be reflected by the increased time spent on the first trial. Nevertheless, this room switch did not change performance levels in the maze, nor did it produce increased activation in cortical control sites, showing that this manipulation did not produce global changes. Likewise, any increases in stress with a switch to a novel room are presumed to be very minor because neither performance in the maze nor behavioral indicators (e.g., fecal boli) were affected by this manipulation.

The dissociation between the dorsal and ventral hippocampus in Experiment 2 supports findings by Moser et al. (1993, 1995) showing that dorsal hippocampal lesions impair performance on a water-maze task, whereas equally sized ventral lesions do not. This functional difference between dorsal and ventral hippocampus is supported by differences in their anatomical connectivity (Witter et al., 1989; Moser and Moser, 1998b) and by the nature of their place cells, which are fewer in the ventral hippocampus and have larger, less selective place fields (Jung et al., 1994). The differences between the dorsal and ventral subiculum were especially evident, with the dorsal subiculum showing relatively greater activation in both experiments. The results from the present study not only show that all hippocampal subfields participate in this dorsal/ventral difference but also reveal that the differential involvement of the dorsal hippocampus is likely to be especially marked on memory tasks that require the learning of novel spatial landmarks. The standard task that is used when showing dorsal/ventral lesion differences is the Morris water maze, in which rats need to learn the layout of a novel test room to locate a platform (Moser et al., 1993, 1995). In a task modification that is more comparable to our study, the testing involved normal rats, and animals were given selective hippocampal lesions after water maze training (Moser and Moser, 1998a). Our results predict that subsequent removal of the dorsal hippocampus would be highly disruptive because initial encoding of the room cues (then novel) involves activity-dependent changes in the dorsal hippocampus. The subsequent removal of this region would therefore render the animal unable to use this information in an efficient manner. It should be noted, however, that the ventral hippocampus was activated in both experiments, and so it might also be expected to contribute. Although Moser and Moser (1998a) found the expected dorsal dominance, their results also showed that normal retrieval required the entire dorsal two-thirds of the hippocampus (i.e., parts of the ventral hippocampus). This evidence for a more distributed mode of action in a normal hippocampus during spatial learning (Moser and Moser, 1998a) is 

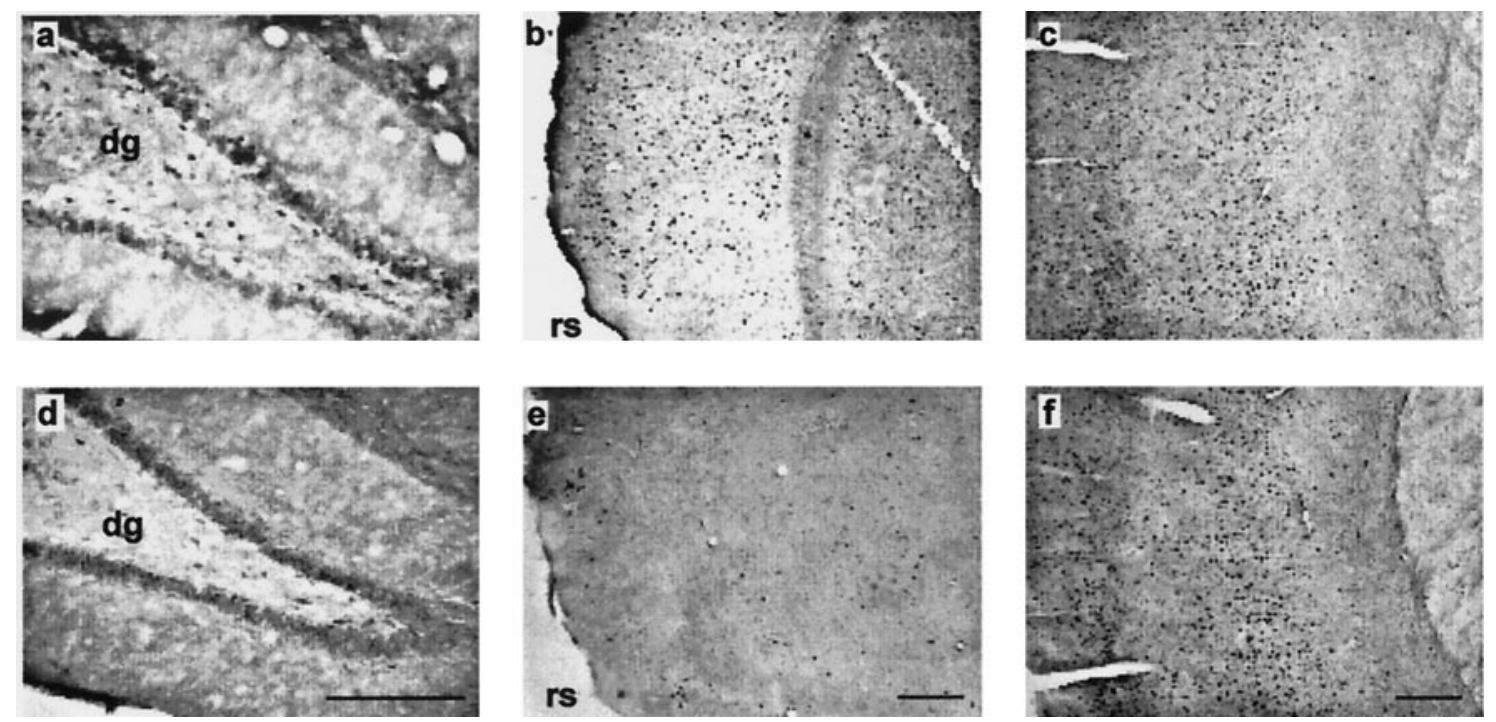

Figure 4. Photomicrographs of matched pairs of coronal sections from Experiment 1 showing Fos-stained nuclei in the dentate gyrus $(a, d)$, postrhinal cortex $(b, e)$, and somatosensory cortex $(c, f)$. The top row shows sections from the experimental condition (Group 8arm-1); the bottom row shows sections from the control condition (Group 1arm). The sections correspond to an AP level of $-2.85(a, d),-7.90(b, e)$, and $-1.53(c, f)$ from bregma (Fig. 1). In the cortical sections $(b, c, e, f)$, the superficial layers are on the left. $d g$, Dentate gyrus; $r s$, rhinal sulcus. Scale bar, $500 \mu \mathrm{m}$.

consistent with our findings. Thus, for spatial memory it may be better to regard the hippocampus as a whole, in that all of the structure can contribute to spatial processing, although the ventral hippocampus is markedly less involved.

Counts of Fos-stained hippocampal nuclei revealed increases across almost all subfields within the hippocampus proper (DG, CA3, CA1, dorsal, and caudal subiculum), indicating that these subfields function as a coherent whole while performing a spatial working memory task. This integrated mode of activity is consistent with the organization of the intrinsic hippocampal connections (Swanson et al., 1987; Amaral and Witter, 1989). Qualitatively similar results were found in a study using 2-deoxyglucose to identify activated brain areas in rhesus monkeys performing a different type of spatial working-memory task (Friedman and Goldman-Rakic, 1988); increased activation was found in DG, CA3, and CA1. Hess et al. (1995b) compared c-fos mRNA levels in separate hippocampal subfields (DG, CA3, and CA1) in rats that had explored a novel environment (training apparatus) or were home-cage controls. They again found an increase in all three subfields in the novel environment animals, with the greatest effect in CA1. This CA1 "dominance" was also found in rats performing a well learned odor discrimination (Hess et al., 1995b). A post hoc analysis of our results revealed that in Experiment 2 there was increased activation in CA1 relative to CA3 when the animals ran the maze in a novel room $(p<0.0001)$. This is consistent with findings that a novel apparatus or novel pattern arrangement also results in increased activation in CA1 (Hess et al., 1995b; Zhu et al., 1997; Wan et al., 1999).

The parahippocampal cortices showed a heterogeneous pattern of c-fos activation. Although the medial entorhinal, lateral entorhinal, and postrhinal cortices all showed increased activation in both experiments, the perirhinal cortex stood out because it did not show significant increases. The perirhinal cortex, however, did show overall the highest level of Fos-stained nuclei within the parahippocampal cortices, indicating a similar involvement across a range of tasks. The qualitatively different pattern of perirhinal activity was underlined by the significant interactions with postrhinal cortex activity and presumably reflects their different connections (Witter et al., 1989). At the same time, the increases in parahippocampal activation in those regions that were responsive were not as great as those found in the hippocampus proper, and it is noteworthy that removal of the postrhinal cortex, and even the entorhinal cortex, can spare performance in tests of spatial memory that are sensitive to hippocampal damage (Kolb et al., 1994; Aggleton et al., 1997; Kesner and Giles, 1998; Bussey et al., 1999; Pouzet et al., 1999).

The perirhinal result was especially striking because this region is densely interconnected with the hippocampus, both directly and indirectly (Witter et al., 1989), yet its lack of differential sensitivity to the spatial tasks revealed clear dissociations with that structure. These dissociations are consistent with previous evidence that exposure to a novel test environment increases hippocampal c-fos activation far greater than perirhinal activation, which did not change significantly (Zhu et al., 1997). Exposure to novel objects or computer-presented visual stimuli produced the opposite pattern of results, i.e., increased perirhinal activation but no hippocampal activation (Zhu et al., 1995, 1996, 1997; Wan et al., 1999). This pattern of differential responsiveness to single elements and complex spatial arrays fits with studies showing that perirhinal cortex lesions disrupt object recognition but have no apparent effect on radial arm maze performance (Mumby and Pinel, 1994; Ennaceur et al., 1996; Ennaceur and Aggleton, 1997; Glenn and Mumby, 1998; Bussey et al., 1999).

Because the perirhinal cortex is activated by discrete novel visual stimuli (Zhu et al., 1996), it seems remarkable that moving a rat to a novel room, which contains novel stimuli, does not produce a clear increase in Fos production. Of relevance, therefore, is the finding that rats shown novel pictures have increased c-fos activity in perirhinal cortex but not hippocampus, yet a spatial rearrangement of familiar pictures (to produce a novel pattern) leads to increased activity in hippocampal subfield CA1 and postrhinal cortex but not in the perirhinal cortex (Wan et al., 1999). This, in turn, can be related to their different patterns of cortical afferents (Witter et al., 1989). Thus novelty of individual items and novelty for the spatial arrangement of items can have quite different consequences. This conclusion is further sup- 
ported by the present interaction between the postrhinal and perirhinal cortices, which not only provides some of the first behavioral evidence for a difference between the postrhinal and perirhinal cortices in spatial working memory but, along with other recent c-fos studies, provides a framework with which to explore these differences further.

\section{REFERENCES}

Aggleton JP, Keen S, Warburton EC, Bussey TJ (1997) Extensive cytotoxic lesions involving both the rhinal cortices and TE impair recognition but spare spatial alternation in the rat. Brain Res Bull 43:279-287.

Amaral DG, Witter MP (1989) The three-dimensional organization of the hippocampal formation: a review of the anatomical data. Neuroscience 31:571-591.

Bertaina V, Destrade C (1995) Differential time courses of c-fos mRNA expression in hippocampal subfields following acquisition and recall testing in mice. Cognit Brain Res 2:269-275.

Burwell RD, Amaral DG (1998) Cortical afferents of the perirhinal, postrhinal, and entorhinal cortices of the rat. J Comp Neurol 398:179-205.

Burwell RD, Witter MP, Amaral DG (1995) Perirhinal and postrhinal cortices of the rat: a review of the neuroanatomical literature and comparison with findings from the monkey brain. Hippocampus 5:390-408.

Bussey TJ, Muir JL, Aggleton JP (1999) Functionally dissociating aspects of event memory: the effects of combined perirhinal and postrhinal cortex lesions on object place memory in the rat. J Neurosci 19:495-502.

Chaudhuri A (1997) Neural activity mapping with inducible transcription factors. NeuroReport 8:3-7.

Dragunow M, Faull R (1989) The use of c-fos as a metabolic marker in neuronal pathway tracing. J Neurosci Methods 29:261-265.

Eichenbaum H, Otto T, Cohen NJ (1994) Two functional components of the hippocampal memory system. Behav Brain Sci 17:449-518.

Ennaceur A, Aggleton JP (1997) The effects of neurotoxic lesions of the perirhinal cortex combined to fornix transection on object recognition memory in the rat. Behav Brain Res 88:181-193.

Ennaceur A, Neave N, Aggleton JP (1996) Neurotoxic lesions of the perirhinal cortex do not mimic the behavioral effects of fornix transection in the rat. Behav Brain Res 80:9-25.

Friedman HR, Goldman-Rakic PS (1988) Activation of the hippocampus and dentate gyrus by working memory: a 2-deoxyglucose study of behaving rhesus monkeys. J Neurosci 8:4693-4706.

Glenn MJ, Mumby DG (1998) Place memory is intact in rats with perirhinal cortex lesions. Behav Neurosci 112:1353-1365.

Herdegen T, Leah JD (1998) Inducible and constitutive transcription factors in the mammalian nervous system: control of gene expression by Jun, Fos and Krox, and CREB/ATF proteins. Brain Res Rev 28:379-490.

Herrera DG, Robertson HA (1996) Activation of c-fos in the brain. Prog Neurobiol 50:83-107.

Hess US, Lynch G, Gall CM (1995a) Changes in c-fos expression in rat brain during odor discrimination learning: differential involvement of hippocampal subfields CA1 and CA3. J Neurosci 15:4786-4795.

Hess US, Lynch G, Gall CM (1995b) Regional patterns of c-fos mRNA expression in rat hippocampus following exploration of a novel environment versus performance of a well learned discrimination. J Neurosci 15:7796-7809.

Hock BJ, Bunsey MD (1998) Differential effects of dorsal and ventral hippocampal lesions. J Neurosci 18:7027-7032.

Jung MW, Wiener SI, McNaughton BL (1994) Comparison of spatial firing characteristics of units in dorsal and ventral hippocampus of the rat. J Neurosci 14:7347-7356.

Kesner RP, Giles R (1998) Neural circuit analysis of spatial working memory: role of pre- and parasubiculum, medial and lateral entorhinal cortex. Hippocampus 8:416-423.
Kolb B, Buhrmann K, McDonald RJ, Sutherland RJ (1994) Dissociation of the medial prefrontal, posterior parietal, and posterior temporal cortex for spatial navigation and recognition memory in the rat. Cereb Cortex 6:664-680.

Morris RGM, Garrud P, Rawlins JNP, O'Keefe J (1982) Place navigation impaired in rats with hippocampal lesions. Nature 297:681-683.

Moser EI, Moser M-B, Andersen P (1993) Spatial learning impairment parallels the magnitude of dorsal hippocampal lesions, but is hardly present following ventral lesions. J Neurosci 13:3916-3925.

Moser M-B, Moser EI (1998a) Distributed encoding and retrieval of spatial memory in the hippocampus. J Neurosci 18:7535-7542.

Moser M-B, Moser EI (1998b) Functional differentiation in the hippocampus. Hippocampus 8:608-619.

Moser M-B, Moser EI, Forrest E, Andersen P, Morris RGM (1995) Spatial learning with a minislab in the dorsal hippocampus. Proc Natl Acad Sci USA 92:9697-9701.

Mumby DG, Pinel JPJ (1994) Rhinal cortex lesions and object recognition in rats. Behav Neurosci 108:11-18.

Naber PA, CaballeroBleda M, JorritsmaByham B, Witter MP (1997) Parallel input to the hippocampal memory system through peri- and postrhinal cortices. NeuroReport 8:2617-2621.

Nagahara AH, Handa RJ (1995) Fetal alcohol exposure alters the induction of immediate early gene mRNA in the rat prefrontal cortex after an alternation task. Alcohol Clin Exp Res 19:1389-1397.

O'Keefe J, Nadel L (1978) The hippocampus as a cognitive map. Oxford, UK: Oxford UP.

Olton DS, Becker JT, Handelmann GE (1979) Hippocampus, space, and memory. Behav Brain Sci 2:313-365.

Olton DS, Walker JA, Gage FH (1978) Hippocampal connections and spatial discrimination. Brain Res 139:295-308.

Otto T, Wolf D, Walsh TJ (1997) Combined lesions of perirhinal and entorhinal cortex impair rats' performance in two versions of the spatially guided radial-arm maze task. Neurobiol Learn Mem 68:28-31.

Pouzet B, Welzl H, Gubler MK, Broerson L, Veenman CL, Feldon J, Rawlins JNP, Yee BK (1999) The effects of NMDA-induced retrohippocampal lesions on performance of four spatial memory tasks known to be sensitive to hippocampal damage in the rat. Eur J Neurosci 11:123-140.

Sagar SM, Sharp FR, Curran T (1988) Expression of c-fos protein in brain: metabolic mapping at the cellular level. Science 240:1328-1331.

Swanson LW (1992) Brain maps: structure of the rat brain. Amsterdam: Elsevier.

Swanson LW, Kohler C, Bjorklund A (1987) The limbic region. I: The septohippocampal system. In: Handbook of chemical neuroanatomy, Vol 5: Integrated systems of the CNS, Part 1 (Bjorklund A, Hokfelt T, Swanson LW, eds), pp 125-277. New York: Elsevier.

Tischmeyer W, Grimm R (1999) Activation of immediate early genes and memory formation. Cell Mol Life Sci 55:564-574.

Wan H, Aggleton JP, Brown MW (1999) Different contributions of the hippocampus and perirhinal cortex to recognition memory. J Neurosci 19:1142-1148.

Wiig KA, Bilkey DK (1994) The effects of perirhinal cortical lesions on spatial reference memory in the rat. Behav Brain Res 63:101-109.

Winer BJ (1971) Statistical principles in experimental design. New York: McGraw-Hill.

Witter MP, Groenewegen HJ, Lopes da Silva FH, Lohman AHM (1989) Functional organization of the extrinsic and intrinsic circuitry of the parahippocampal region. Prog Neurobiol 33:161-253.

Zhu XO, Brown MW, McCabe BJ, Aggleton JP (1995) Effects of novelty or familiarity of visual stimuli on the expression of the immediate early gene c-fos in rat brain. Neuroscience 69:821-829.

Zhu XO, McCabe BJ, Aggleton JP, Brown MW (1996) Mapping visual recognition memory through expression of the immediate early gene c-fos. NeuroReport 7:1871-1875.

Zhu XO, McCabe BJ, Aggleton JP, Brown MW (1997) Differential activation of the rat hippocampus and perirhinal cortex by novel visual stimuli and a novel environment. Neurosci Lett 229:141-143. 